\title{
Finite-size effects in the nuclear magnetic resonance of epitaxial palladium thin films
}

\author{
W. A. MacFarlane, ${ }^{1,}{ }^{*}$ T. J. Parolin, ${ }^{1}$ T. I. Larkin, ${ }^{2}$ G. Richter, ${ }^{3}$ K. H. Chow, ${ }^{4}$ M. D. Hossain, ${ }^{5}$ R. F. Kiefl,,${ }^{5,6}$ C. D. P. Levy, ${ }^{6}$ \\ G. D. Morris, ${ }^{6}$ O. Ofer, ${ }^{6}$ M. R. Pearson, ${ }^{6}$ H. Saadaoui, ${ }^{7}$ Q. Song, ${ }^{5}$ and D. Wang ${ }^{5}$ \\ ${ }^{1}$ Department of Chemistry, University of British Columbia, Vancouver, British Columbia, V6T IZ1 Canada \\ ${ }^{2}$ Max-Planck-Institut für Festkörperforschung, D-70569 Stuttgart, Germany \\ ${ }^{3}$ Max-Planck-Institut für Intelligente Systeme, D-70569 Stuttgart, Germany \\ ${ }^{4}$ Department of Physics, University of Alberta, Edmonton, Alberta, T6G 2 El Canada \\ ${ }^{5}$ Department of Physics and Astronomy, University of British Columbia, Vancouver, British Columbia V6T 1Z1 Canada \\ ${ }^{6}$ TRIUMF, 4004 Wesbrook Mall, Vancouver, British Columbia, V6T 2A3 Canada \\ ${ }^{7}$ Paul Scherrer Institute, Laboratory for Muon Spin Spectroscopy, 5232 Villigen PSI, Switzerland \\ (Received 9 August 2013; revised manuscript received 10 October 2013; published 28 October 2013)
}

\begin{abstract}
We have measured the NMR of ${ }^{8} \mathrm{Li}^{+}$implanted in a set of thin epitaxial films of Pd. We find a large, negative, strongly temperature-dependent Knight shift $K$ consistent with previous measurements on polycrystalline films. The temperature dependence of the shift exhibits a characteristic deviation from the susceptibility $\chi(T)$. In particular, at low temperature, $K(T)$ continues to follow a simple Curie-Weiss dependence. This result provides important insight into the origin of the low-temperature behavior of $\chi(T)$ in strongly paramagnetic metals. In addition, we find the room temperature shift depends on film thickness, with changes on the order of $20 \%$ between films $100 \mathrm{~nm}$ and $30 \mathrm{~nm}$ thick. We also observe a surface-related resonance in both Au-capped and uncapped films with a small positive shift. These features bear a striking similarity to the Pt NMR line shapes in much smaller Pt particles. However, they seem to originate, not from adsorbed species, but rather in confinement effects on the highly exhange-enhanced $\mathrm{Pd} d$ band.
\end{abstract}

DOI: 10.1103/PhysRevB.88.144424

PACS number(s): 76.60.-k, 75.20.En, 75.70.Ak

\section{INTRODUCTION}

The Knight shift of ${ }^{8} \mathrm{Li}^{+}$in metallic Pd is the largest known among paramagnetic metals. In NMR, the Knight shift provides a sensitive local measurement of the spin susceptibility of the conduction band, which for Pd is significantly temperature dependent, reflecting Pd's proximity to ferromagnetism. ${ }^{1}$ Here we measure the NMR using the radioactive $\beta$ decay in a special technique known as $\beta$-detected NMR ( $\beta$-NMR). ${ }^{2}$ In $\beta$-NMR the radioactive probe atom is generally extrinsic to the metal, but the coupling between the nuclear spin and the surrounding conduction electrons is analogous to intrinsic NMR, and, in most cases, yields a similar result. ${ }^{3}$ The advantage of $\beta$-NMR is to allow such measurements in circumstances where the conventional NMR cannot be done. For example, in metals for which there is no favorable NMR nucleus. Pd is such a case as its nuclear moment is small, and there are consequently very few reports of ${ }^{105} \mathrm{Pd} \mathrm{NMR} .{ }^{4,5}$ Another example is the case of a thin film heterostructure where there are too few nuclei for conventional NMR to be practical.

In this paper, we report $\beta$-NMR measurements of the ${ }^{8} \mathrm{Li}$ Knight shift in high-quality epitaxial thin films of $\mathrm{Pd}$ grown on $\mathrm{SrTiO}_{3}$ (STO) substrates. We find a large negative and temperature-dependent Knight shift consistent with our previous measurements in polycrystalline films, ${ }^{6,7}$ but the high film quality results in narrower resonances and the improved data yield more accurate and reliable shifts. Our three main findings are: (i) We confirm the temperature-dependent shift does not simply scale with the susceptibility $\chi(T)$ of $\mathrm{Pd}$ at low temperatures, ${ }^{6}$ rather it scales well with the Curie-Weiss (C-W) law that characterizes $\chi$ at high temperature. ${ }^{8}$ (ii) $\mathrm{We}$ find a significant dependence of the shift on film thickness in the range $30-100 \mathrm{~nm}$ : the thickest film is very similar to bulk $\mathrm{Pd}$, while in the thinner films, the shift is substantially reduced, possibly due to quantum confinement and surface modification of the electronic structure of the films. (iii) $\mathrm{We}$ find another ${ }^{8} \mathrm{Li}$ resonance with a small positive shift associated with the near-surface region of the films. This resonance likely originates from metallic Pd near the surface where the $d$-band susceptibility has been strongly suppressed, but a small $s$-band Knight shift remains. The spectra, therefore, show a remarkable similarity to the ${ }^{195} \mathrm{Pt}$ NMR in small platinum particles. ${ }^{9-12}$ However, the model usually invoked to explain such spectra cannot quantitatively account for our results without a substantially larger healing length.

\section{EXPERIMENT}

\section{A. Samples}

The samples used in this work are four epitaxial thin films on $8 \times 10 \mathrm{~mm}\langle 100\rangle$ STO substrates (Crystal GmbH). While STO is closely lattice matched to Pd, the epitaxy is not simple, but is carried out in a well-established three-step process. ${ }^{13}$ The $99.95 \%$ Pd source was the same for all the films and the growths were done in the MBE system used in Ref. 13 with a typical background pressure of $5 \times 10^{-10}$ Torr. This results in epitaxial orientation of the $\mathrm{Pd}$ overlayer with alignment of the cubic axes, $\langle 100\rangle_{\mathrm{Pd}} \|\langle 100\rangle_{\mathrm{STO}}$ both in and out of the plane of the film. Two of the Pd films were capped in situ at room temperature with $(99.99 \%) \mathrm{Au}$ at $0.02 \mathrm{~nm} / \mathrm{s}$ to protect the Pd surface from contamination by atmospheric adsorbates and to assess any role for such contamination. Under these conditions, the Au may also exhibit some epitaxy with the underlying Pd. ${ }^{14}$ To minimize systematic differences, the films were grown simultaneously in pairs (I, II and III, IV) using a shutter to interrupt deposition. The sample characteristics are summarized in Table I. Sample IV is dimensionally similar to 
TABLE I. Nominal and ellipsometrically determined $\left(\mathrm{Pd}_{\mathrm{ell}}, \mathrm{Au}_{\mathrm{ell}}\right)$ layer thicknesses of the Pd heterostructures.

\begin{tabular}{lcccc}
\hline \hline$\#$ & $\mathrm{Pd}(\mathrm{nm})$ & $\mathrm{Au}(\mathrm{nm})$ & $\mathrm{Pd}_{\text {ell }}(\mathrm{nm})$ & $\mathrm{Au}_{\text {ell }}(\mathrm{nm})$ \\
\hline $\mathrm{I}$ & 30 & 0 & 29.8 & - \\
II & 30 & 7.5 & 24 & 5.4 \\
III & 50 & 0 & $46-47$ & - \\
IV & 100 & 7.5 & thick & 8.5 \\
\hline \hline
\end{tabular}

the polycrystalline $e$-beam evaporated sample used in Ref. 6 . These two samples are also similar in nominal purity, but the MBE growth process is much cleaner and the crystallinity of the resulting films is much higher. Most of the measurements reported here were taken on sample IV.

Additionally the samples were characterized using variable wavelength (245-1680 nm) optical ellipsometry at room temperature, using a Woollam M2000 ellipsometer in air. The resulting data were fit with a model of the heterostructures using literature values of the optical constants of Pd, Au and STO. In addition, an intermixed $\mathrm{Au} / \mathrm{Pd}$ layer (in an effective medium approximation) was considered. From least-squares fitting, there was no evidence for intermixing, but the model indicates no sensitivity to an intermixed layer less than $1 \mathrm{~nm}$ thick.

In modeling the ellipsometric data, we also considered models involving contamination by atmospheric species, since the samples are exposed to ambient atmosphere between fabrication and measurement. Small adsorbate molecules and oxide supports have significant effects on the NMR of small Pt particles. ${ }^{9,11}$ Like Pt, Pd has a significant tendency to adsorb atmospheric molecules. In addition, $\mathrm{Pd}$ is well known for its ability to absorb hydrogen, and at sufficient concentration, interstitial $\mathrm{H}$ causes significant reduction in $\chi$ by doping. ${ }^{15}$ However, $\mathrm{H}$ absorption is easily reversible in vacuum at room temperature, ${ }^{17}$ so an ex situ measurement of $\mathrm{H}$ content provides only an upper limit to contamination during the measurement. For these reasons, we considered a $\mathrm{PdO}$ oxide overlayer ${ }^{16}$ on the bare films, and interstitial hydride contamination, i.e., $\mathrm{PdH}_{x}$ with optical properties modeled using an effective medium mixture of pure $\mathrm{Pd}$ and $\mathrm{PdH}_{1} \cdot{ }^{17}$

We find no evidence for an oxide layer, rather the model indicates an oxide or degraded surface layer must be less than $1 \mathrm{~nm}$ thick if it exists at all. Similarly there is no evidence for hydride contamination, and no amount of hydride is found to improve the fit. Besides confirming the material purity of the samples, the model allows us to extract layer thicknesses (except for the $100 \mathrm{~nm} \mathrm{Pd} \mathrm{film,} \mathrm{which} \mathrm{is} \mathrm{optically} \mathrm{thick),}$ and the best fit values, $\mathrm{Pd}_{\text {ell }}$ and $\mathrm{Au}_{\text {ell }}$, are reported in Table I. From the fits, the uncertainty in the values is in the last reported digit, but a larger systematic uncertainty arises from uncertainty in the wavelength-dependent complex indices of refraction that are used as inputs to the model. Comparing samples, the best fit was obtained for IV and the worst for III. These values represent the average properties over the optical beam spot, which was about $4 \times 6 \mathrm{~mm}$ and roughly centered on the films. A systematic discrepancy with the nominal thicknesses is expected due to the physical offset of the quartz crystal microbalance that monitors the growth in the MBE flux.

\section{B. Spectroscopy}

$\beta$-NMR resonance measurements were performed by implanting highly spin polarized ${ }^{8} \mathrm{Li}^{+}$at energies ranging from 1.5 to $28 \mathrm{keV}$ at TRIUMF's ISAC polarized beamline. The ${ }^{8} \mathrm{Li}$ nucleus is spin $I=2$, with gyromagnetic ratio $\gamma=$ $6.3015 \mathrm{MHz} / \mathrm{T}$ and electric quadrupole moment $Q=$ $+31.4 \mathrm{mb}$, and is polarized by in-flight optical pumping to polarization $\sim 70 \%$. Two distinct types of resonance measurement were done: continuous wave $(\mathrm{CW})$ resonances where the transverse radio frequency (RF) magnetic field is applied as a continuous sine wave with frequency stepped slowly relative to the ${ }^{8} \mathrm{Li}$ lifetime of $1.21 \mathrm{~s}$ through a frequency range around the nuclear Larmor frequency. This mode of measurement is advantageous for lines wider than a few $\mathrm{kHz}$, but the resulting line shape may be broadened by the RF field (power broadening) and dynamical processes such as spin diffusion. The power broadening can be reduced by lowering the $\mathrm{CW}$ $\mathrm{RF}$ amplitude, but at the expense of signal amplitude. We have recently developed a pulsed RF mode to eliminate or reduce such nonintrinsic broadening. ${ }^{18}$ In this mode, which also uses a continuous ion beam, modulated RF pulses that address sharply defined frequency regions are arranged to uniformly cover a range of frequencies. The pulses are then processed in random order to limit hysteretic effects, and the signal is extracted from the step in the decay asymmetry caused by the RF pulse. Due to the limited RF field available in the spectrometer, this type of measurement is much more time consuming especially for lines wider than a few $\mathrm{kHz}$. In the absence of dynamical effects, the pulsed RF spectrum should match the low power limit of the CW spectrum.

The samples were all run within a few weeks of fabrication. They were stored under vacuum, but during mounting are exposed to ambient atmosphere for several minutes. Resonances were measured in a homogeneous static applied field of $4.1 \mathrm{~T}$ perpendicular to the surface of the film. The ${ }^{8} \mathrm{Li}^{+}$ion beam implantation energy was typically $13 \mathrm{keV}$. The beam intensity was on the order of $10^{6}$ ions per second into a beam spot $2-3 \mathrm{~mm}$ diameter centered on the sample. As a frequency calibration the resonance in a single crystal of $\mathrm{MgO}$ was measured both before and after the Pd films with the superconducting solenoid in persistence mode. The reference frequency in $\mathrm{MgO}$ was found to be 25834808 (5) $\mathrm{Hz}$, with no evidence of drift during the course of the experiment. Finally, sample IV was remeasured at a lower field of 2.2 Tesla to confirm that the shift is paramagnetic, i.e., scales with the applied field.

\section{Probe ion implantation}

In the experiments, we can decelerate the ion beam by biasing the spectrometer (including the sample) at a positive voltage. Thus we can vary the implantation energy from the beam energy (typically $20-30 \mathrm{keV}$ ) down to as low as $100 \mathrm{~s}$ of $\mathrm{eV}$. This allows us to probe the sample in a depth-resolved manner. The decelerating potential affects the ion beam focusing, so it is usually necessary, at each energy, to adapt the incident ion beam tune to obtain a well-focused beam spot on the sample. At very low energies, the signal diminishes in amplitude, in part, because a fraction of the ions backscatter rather than implant, making measurements 


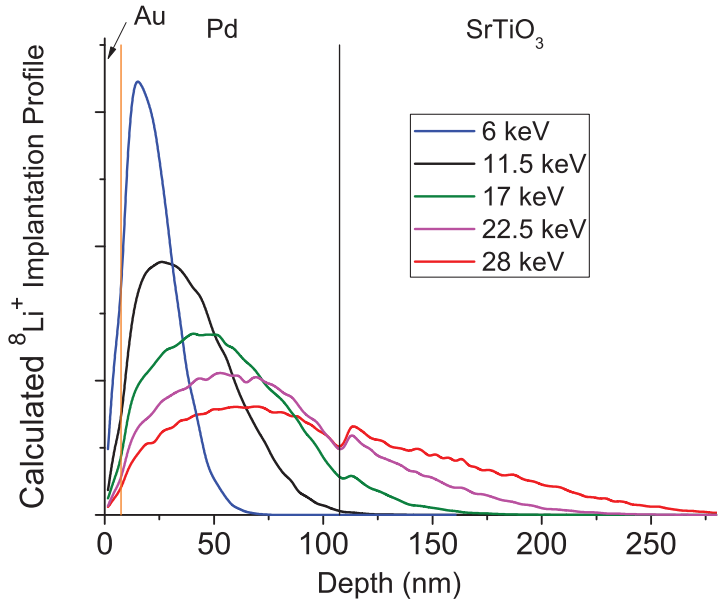

FIG. 1. (Color online) Predicted implantation profile of ${ }^{8} \mathrm{Li}^{+}$in sample IV for several representative energies from the Monte Carlo code TRIM.SP. The vertical lines indicate heterointerfaces.

at very low energies more difficult. For these reasons, we investigate a reduced range of implantation energies where such backscattering is negligible.

Ion implantation in solids is a random process that can be simulated quite accurately using Monte Carlo methods. These codes account for the composition and density of the stopping material but not for its crystal structure, so they do not include effects such as channeling. Using the TRIM.SP code, ${ }^{19}$ we calculated the implantation profile of ${ }^{8} \mathrm{Li}^{+}$at various energies in the accessible range. Results for sample IV, shown in Fig. 1, reveal that, at the implantation energies used here, the signal is dominated by the Pd film, but at the lowest energies, we expect a contribution from the Au overlayer as well. At the full energy, a significant fraction of the ${ }^{8} \mathrm{Li}$ penetrates the film and stops in the substrate. The $\beta$-NMR in STO consists of small quadrupole satellites split by more than $100 \mathrm{kHz}$ from the Larmor frequency. ${ }^{20}$ These satellites are well outside the frequency range of the spectra here, so we do not observe the signal from the substrate. From the predicted profiles, we can extract the mean range for the probe ion as a function of implantation energy. However, the implantation profiles are significantly broadened by range straggling.

Implantation does cause damage to the host lattice. Frenkel (vacancy-interstitial) pairs are the main defect produced by collisions of the incident ion with atoms of the host lattice, while electronic excitations relax very quickly on the time scale of the measurement. Because the ${ }^{8} \mathrm{Li}^{+}$is only about $10 \%$ the mass of the host Pd atoms, they are quite ineffective at producing vacancies. For example, Monte Carlo calculations predict that only about $1 \%$ of the ${ }^{8} \mathrm{Li}^{+}$energy loss corresponds to vacancy formation. The implanted ion generally stops at a high symmetry crystallographic site some distance away from the final long-lived implantation-related defect. In many cases, in elemental metals, we find narrow resonances near the Larmor frequency, indicating a highly ordered local environment at the stopping site with no evidence of implantation damage, e.g., Ref. 21. This implies the distance of the stopped probe to the nearest implantation damage is sufficient that it doesn't affect the NMR either magnetically or through the quadrupolar interaction. The minimum distance for this is material dependent, but may be only a few lattice constants. However, a fraction of the probe ions may stop in the vicinity of implantation damage. The ensemble of configurations of probe and nearby damage would produce a broad resonance due to a random distribution of quadrupolar splittings as has been seen, for example, in the $\beta$-NMR of ${ }^{12} \mathrm{~B}$ implanted in the semiconductor ZnSe. ${ }^{22}$ Our sensitivity to broad resonances diminishes for lines wider than $10-20 \mathrm{kHz}$, due to the limited RF power, so the occurrence of such sites may only be detected as a reduced amplitude of the signal from ordered sites.

Finally, because the total fluence of probe ions into the sample over the entire experiment is much lower than the number of host atoms in the sampled volume, we find no evidence of cumulative damage to the sample in the form of a rate dependence or a systematic evolution of the signal. Moreover, the ${ }^{8} \mathrm{Be}$ daughter of the $\beta$ decay is very short lived, decomposing promptly into two $\alpha$ particles, leaving no longlived dopants in the sample.

\section{RESULTS}

The high field CW $\beta$-NMR spectra are qualitatively similar to previous measurements in polycrystalline Pd films and bulk foil: ${ }^{6,23}$ the Pd resonance is strongly negatively shifted with respect to the $\mathrm{MgO}$ reference, and the line shape is noticeably asymmetric with a long tail extending towards zero shift. Figure 2 shows a subset of the spectra for Au-capped sample IV as a function of temperature. At $13 \mathrm{keV}$ implantation energy, the spectrum consists of two well-separated lines labeled Pd (low frequency) and one slightly positively shifted relative to $\mathrm{MgO}$, labeled "surface $+\mathrm{Au}$ ". By reducing the implantation energy, the latter resonance is enhanced, confirming its origin near the sample surface. The shift of ${ }^{8} \mathrm{Li}$ in gold is known to be small and positive, ${ }^{21}$ further confirming its assignment to the Au cap. However, both its amplitude and width appear too large to be consistent with the thin Au overlayer. At room

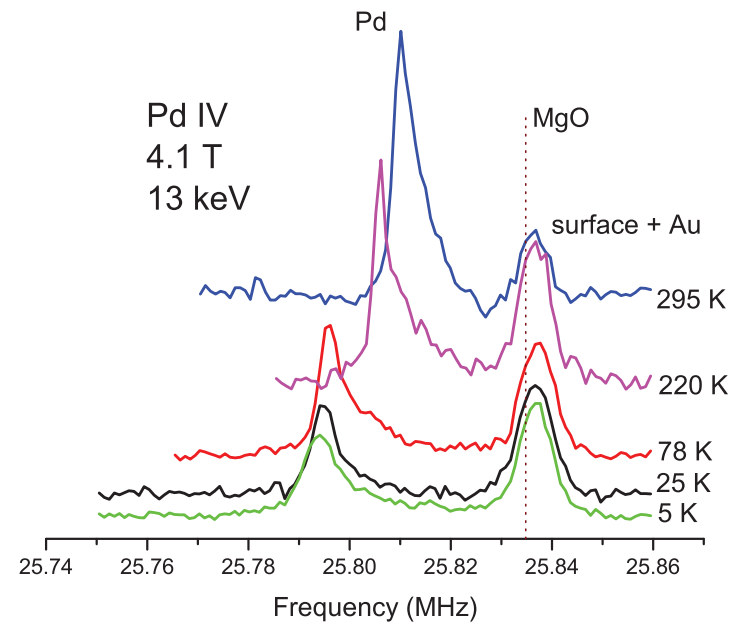

FIG. 2. (Color online) CW resonance spectra at selected temperatures in sample IV at $4.1 \mathrm{~T}$ using an ${ }^{8} \mathrm{Li}^{+}$implantation energy of $13 \mathrm{keV}$. The high-frequency line to the right is from the Au-capping layer as well as the Pd near the surface, while the lower-frequency line is that of Pd deeper in the film. The vertical dashed line marks the resonance frequency in $\mathrm{MgO}$, which we use conventionally as the zero of the shift scale. 


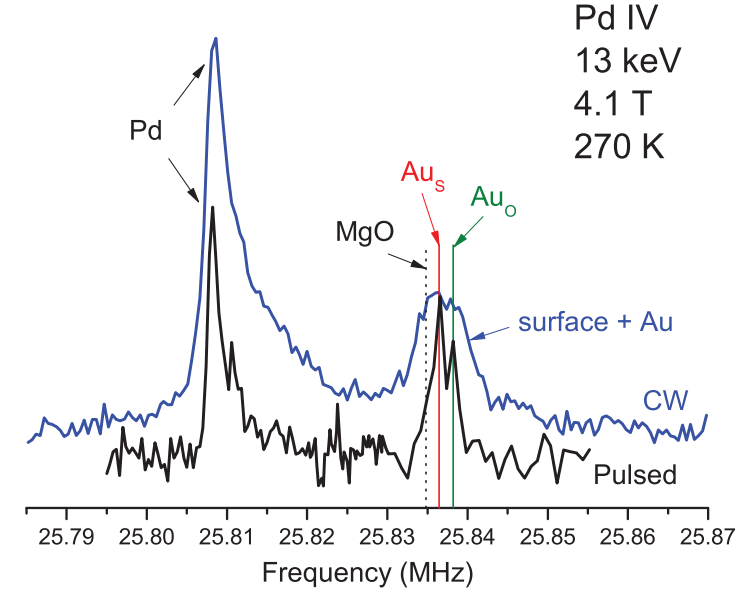

FIG. 3. (Color online) Comparison of pulsed and $\mathrm{CW}$ resonance spectra at $270 \mathrm{~K}$ in sample IV at 4.1 Tesla using an ${ }^{8} \mathrm{Li}^{+}$implantation energy of $13 \mathrm{keV}$. The signal to noise is much lower in the pulsed case, but the resolution is much higher yielding a more clearly defined value for the shifts. The long high-frequency tail in the $\mathrm{CW}$ spectrum is highly suppressed in the pulsed spectrum. The composite resonance at small positive shift matches very well to the known shifts of ${ }^{8} \mathrm{Li}^{+}$ in $\mathrm{Au}^{21}$ (vertical lines, labeled $\mathrm{Au}_{O}$ and $\mathrm{Au}_{S}$ ), but the corresponding resonance in the $\mathrm{CW}$ spectrum (labeled "surface $+\mathrm{Au}$ ") is too large and too wide to be due simply to the Au overlayer.

temperature, the Pd resonance is substantially narrower than in previous measurements on polycrystalline films ${ }^{6,7}$ (by about a factor of two), confirming the higher quality of the current samples and allowing more refined measurements.

We also acquired pulsed RF spectra. In such high-resolution spectra, the amplitudes of lines broader than a few $\mathrm{kHz}$ are strongly suppressed. ${ }^{18}$ Figure 3 shows an example for comparison. Under the same conditions, the $\mathrm{CW}$ spectrum took 20 minutes to acquire, while the pulsed spectrum took over 70 minutes. The Pd resonance shows a pronounced asymmetric broadening towards zero shift. As expected this broad contribution is largely suppressed in the pulsed RF spectrum. This characteristic asymmetric line shape seems to be intrinsic to $\mathrm{Pd}$, as it was observed in other samples. Its origin is not clear, but we discuss some possibilities below in Sec. V A.

In contrast, two sharp lines at small positive shift are expected for the $\mathrm{Au}$ at this temperature, ${ }^{21}$ corresponding to two different cubic sites (the octahedral interstitial $\mathrm{Au}_{O}$, and the substitutional $\mathrm{Au}_{S}$ ). The excellent match of the frequencies of the known shifts in $\mathrm{Au}(+63$ and $+131 \mathrm{ppm}$, vertical lines in Fig. 3) is a strong confirmation that they are from the $\mathrm{Au}$ overlayer, though the $\mathrm{Au}_{O}$ line is larger than expected at this temperature. ${ }^{21}$ The corresponding line in the $\mathrm{CW}$ spectra is substantially wider than would be expected for Au, suggesting that there is another superimposed broad resonance that is largely suppressed in the pulsed spectra. This is confirmed by the presence of a similar line in the CW spectra of the uncapped Pd films (see below, Fig. 5). We label this line the "surface" line, but we note a negligible fraction of the probes reside in the top atomic layer, so in fact this resonance corresponds to a thin layer of Pd near the surface in both capped and uncapped films. With the present data, the thickness of this layer is difficult to determine accurately, but it is on the order of $10 \mathrm{~nm}$.
To extract the Pd shift, the pulsed spectra were fit to a Gaussian line. We note that this is a different convention than was used previously for CW spectra. ${ }^{6,7}$ The CW Pd spectra were fit to a narrow Gaussian and broad Lorentzian line to account for the high-frequency tail. The position of the latter was fixed to a constant fraction of the shift of the sharp line (details in the next section). We consider this line shape a reasonable phenomenological choice that adequately describes the CW spectra. From these fits, the Pd shift was determined from the position of the narrow resonance which coincides with that of the pulsed spectrum (Fig. 3).

From the resonance positions in the fits, the relative shift is

$$
K=\frac{v(\mathrm{Pd})-v(\mathrm{MgO})}{v(\mathrm{MgO})},
$$

which is plotted as a function of temperature in Fig. 4. This figure clearly demonstrates that the two data sets and fits give consistent results, which further indicates the robustness of the results. The resonances broaden somewhat at low temperature with an accompanying reduction in amplitude that keeps the overall area approximately constant below $200 \mathrm{~K}$. The broadening is smaller than previous samples, consistent with a higher degree of microscopic homogeneity. The most accurate measurements of the shift can be made with the relatively large narrow resonance near room temperature. The dependence on film thickness was thus investigated at $295 \mathrm{~K}$.

Figure 5 shows comparable $\mathrm{CW}$ spectra in all four samples. In the raw data, there is a clear sample dependence of the Pd resonance in both the line position and shape, with the resonance in the thinner films being less negatively shifted. The spectrum is broadest in the uncapped $50 \mathrm{~nm}$ film. The two $30 \mathrm{~nm}$ films have very similar spectra, indicating the change in shift is unrelated to atmospheric adsorbates or oxidation that would be prevented by the Au capping. To confirm this,

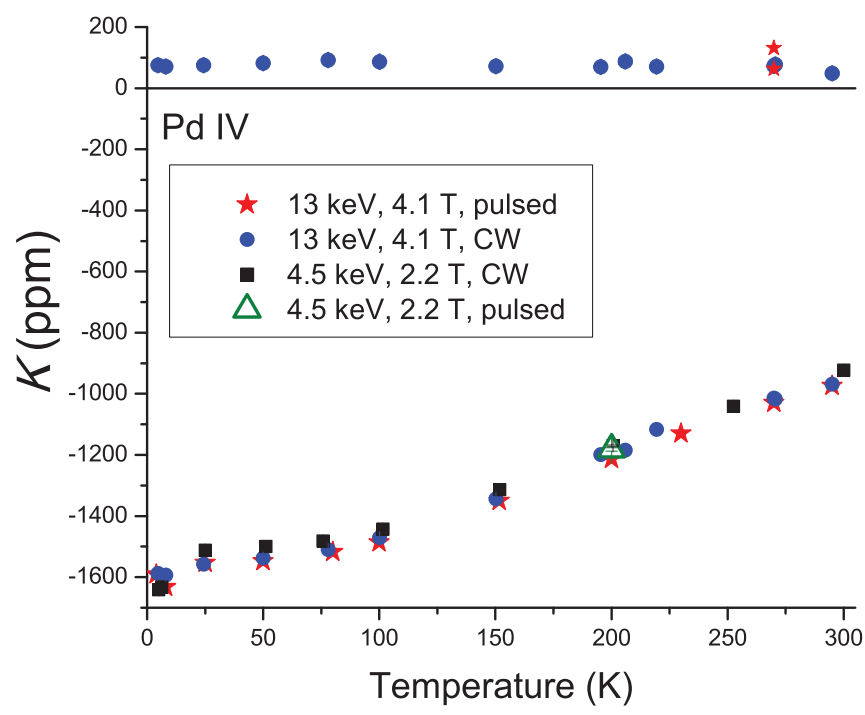

FIG. 4. (Color online) The relative shift of the resonance with respect to $\mathrm{MgO}$ as a function of temperature for Pd sample IV at $4.1 \mathrm{~T}$ and $13 \mathrm{keV}$ implantation energy (2009 data) and at 2.2 Tesla and $4.5 \mathrm{keV}$ (2012 data). The consistency of the two fields confirms that the shift is magnetic. The temperature dependence is due to the magnetic susceptibility of Pd. 


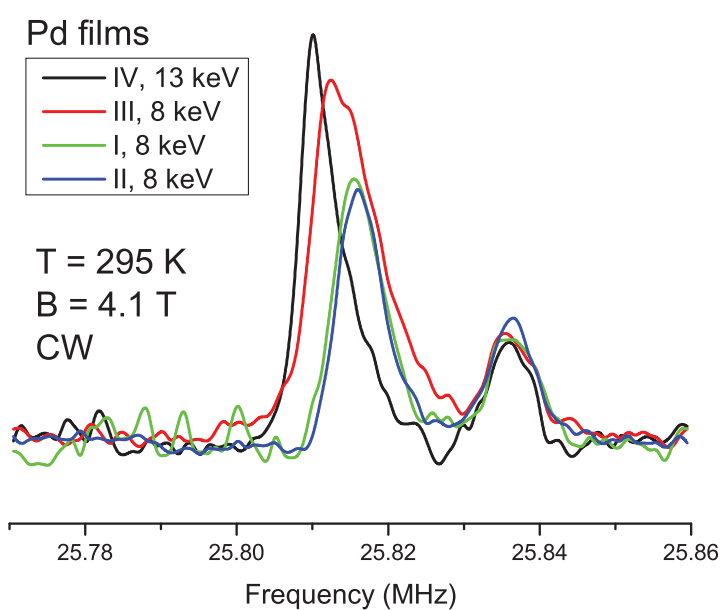

FIG. 5. (Color online) $\mathrm{CW}{ }^{8} \mathrm{Li}^{+} \beta$-NMR spectra in all four samples at $295 \mathrm{~K}$ demonstrating the thickness dependence of the shift of ${ }^{8} \mathrm{Li}^{+}$in Pd films. The high-frequency resonance is present in both capped and uncapped Pd films with a similar amplitude. This line is enhanced when the implantation energy is reduced. The shift of the low-frequency Pd resonance is systematically reduced in the thinner films.

we also acquired pulsed resonance spectra under the same conditions. Though these spectra are significantly noisier, they are consistent, including the substantial broadening of the resonances for the thinner films, compared to IV. This trend towards lower shift is also consistent with what we have observed in other thin polycrystalline Pd films. ${ }^{24}$

In contrast, the surface resonance is similar to sample IV in all the films (capped and uncapped), with a small positive and relatively temperature-independent shift of about $+75 \mathrm{ppm}$. The small positive shift is similar to the ${ }^{8} \mathrm{Li}^{+}$Knight shift in $\mathrm{Au}, \mathrm{Ag}$, and Cu. ${ }^{21}$ However, for the Au-capped samples, based on the predicted implantation profiles, its amplitude is too large to be due solely to the capping layer. For the two $30 \mathrm{~nm}$ films, this resonance is larger in the Au-capped film, consistent with an additional contribution due to the Au overlayer. This surface resonance was also observed in previous measurements in the polycrystalline film, ${ }^{6}$ where it was attributed to the $\mathrm{Au}$ overlayer, but there too it was too large and broad to be due solely to the Au.

As described above, we fit these spectra to obtain the resonance shift from the sharp peak and low-frequency edge of the resonance. At $295 \mathrm{~K}$, the relative shift is presented below in Sec. V B, in comparison with earlier measurements on other samples.

\section{ANALYSIS OF THE SHIFT}

In this section, we consider the temperature dependence of the Knight shift of ${ }^{8} \mathrm{Li}^{+}$in Pd sample IV. The fits yield the raw shifts $K(T)$. The consistency of the results from the pulsed and CW spectra fit with different line shapes indicates the reliability of the extracted shifts, while the consistency between the 4.1 and $2.2 \mathrm{~T}$ values is clear confirmation that the shift is magnetic (not quadrupolar) and that its temperature dependence is not field dependent, i.e., that it is due to paramagnetism of Pd with no evidence of metamagnetism.

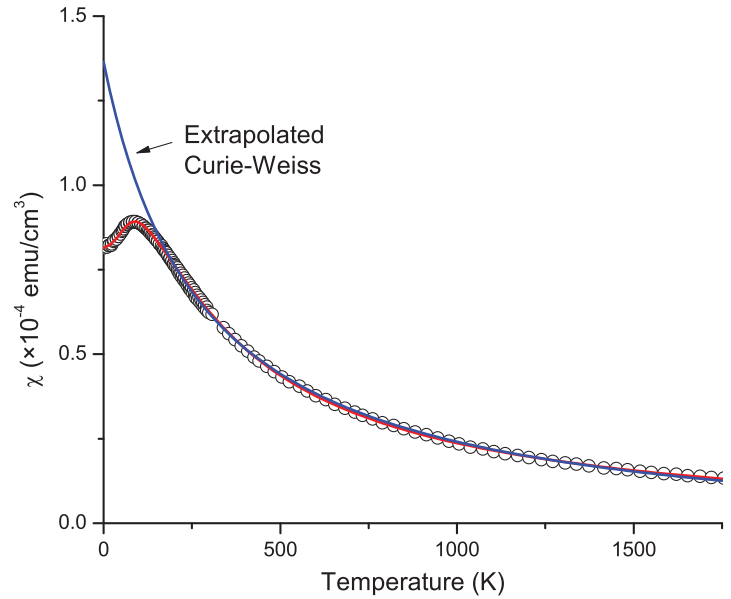

FIG. 6. (Color online) The magnetic susceptibility of Pd from the literature ${ }^{26}$ together with a phenomenological fit used in correcting the shift measurements for the demagnetizing field (red curve) and the extrapolated high-temperature Curie-Weiss dependence (blue curve).

Indeed the largest effect of the reduced field is the lower resolution.

To account for the demagnetization field $B_{d}$, which results in a magnetic shift $K_{d}$, present within the Pd film, ${ }^{25}$ we require its uniform static magnetic susceptibility, since

$$
K_{d}=\frac{8 \pi}{3} \chi
$$

independent of film thickness, where $\chi$ is the cgs volume susceptibility, and the demagnetization-corrected shift is $K_{c}=$ $K+K_{d}$. Equation (2) is based on the demagnetization factor for a thin uniform sheet in a perpendicular field. As the films are not sufficiently massive to measure $\chi$, even in a sensitive magnetometer, we choose literature values. ${ }^{26}$ This $\chi(T)$ is shown in Fig. 6 together with a phenomenological parametrization (accurate to better than $1 \%$ over the relevant $T$ range). We use this (red curve in Fig. 6) to calculate $K_{d}$. Recalling that the demagnetization field is a classical contribution to the internal field in a magnetized body that represents the field due to the uniformly magnetized continuum outside a Lorentz sphere centered on the probe, and defined such that material within the sphere must be treated atomically. ${ }^{8,25}$ It is thus appropriate to use the bulk susceptibility to calculate $K_{d}$, provided $\chi$ is not substantially different from the bulk. As will be shown below, this is evidently the case for the $100 \mathrm{~nm}$ film. With this assumption, the demagnetization field is seen to account for about half of the overall shift, so the corrected shift, while still negative, is substantially smaller.

The corrected shift results from a different component of the local internal field experienced by the ${ }^{8} \mathrm{Li}$ probe nuclei, namely the hyperfine contact field, and it represents the Knight shift that is expected to be proportional to the electronic spin susceptibility, i.e.,

$$
K_{c}=A \chi .
$$

Unlike the NMR of transition metals, the ${ }^{8} \mathrm{Li}$ hyperfine coupling is much smaller, so $K_{d}$ is a substantial part of the overall shift. ${ }^{27}$ The effect of correcting for demagnetization is shown in Fig. 7. The blue curve represents $K_{d}$ and is 


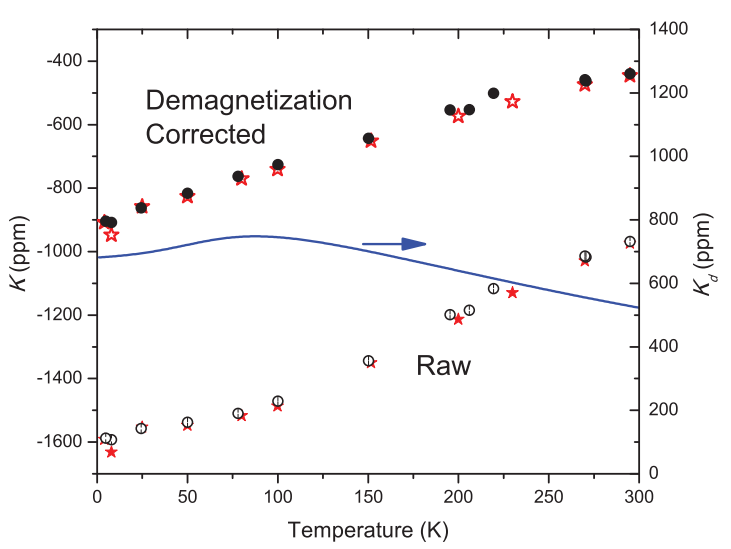

FIG. 7. (Color online) The effect of demagnetization correction on the shift in sample IV. The raw shifts from Fig. 4 are replotted, with the demagnetization correction, a scaling of the susceptibility shown in Fig. 6 (blue curve, right scale). The stars are the pulsed and the circles the $\mathrm{CW}$ shifts. The correction results in the smaller negative shifts shown. The curvature of the raw shift is largely removed by the correction.

just a scaled version of $\chi(T)$ from Fig. 6 over the reduced temperature range.

Since $K_{d}$ is a function of temperature, the temperature dependence of the corrected shift differs from the raw shift (see Fig. 7). It is clear from the data that the two contributions to the shift (demagnetization and hyperfine field) do not have the same temperature dependence. Remarkably the corrected shift exhibits a very smooth almost linear temperature dependence. For comparison, we include the shifts from the previous measurement on a polycrystalline film ${ }^{6}$ with the shift extracted and corrected in exactly the same manner, see Sec. V C below.

For the fits of the Pd spectra, we accounted for the high frequency tail (e.g., Fig. 2) by fitting a second broad, unresolved Lorentzian whose position is fixed by assuming a hyperfine coupling 0.92 that of the sharp resonance, so its demagnetization corrected shift is

$$
K_{c}^{L}=0.92 K_{c} \text {. }
$$

Applying this assumption required calculation of the demagnetization field at the stage of fitting, but it yielded excellent fits to the asymmetric Pd spectra at both fields and all temperatures.

\section{DISCUSSION}

\section{A. ${ }^{8} \mathbf{L i}$ line shape in $\mathbf{P d}$}

We begin by discussing the ${ }^{8} \mathrm{Li}^{+}$shape of the negatively shifted $\mathrm{Pd}$ resonance. The asymmetric line shape is consistent with a polycrystalline film ${ }^{6}$ and bulk foil. ${ }^{23}$ This similarity strongly suggests the shape is intrinsic, though the high-frequency tail is somewhat sample dependent, possibly indicating a sensitivity to crystalline purity and order. The present samples have a higher degree of order and clearly still exhibit this asymmetric line. In this section, we discuss possible origins of this line shape.

First, we rule out quadrupolar coupling as the origin of the shape of the $\mathrm{Pd}$ resonance. For the $I=2^{8} \mathrm{Li}$ nucleus, the quadrupolar satellite pattern is a quartet of lines centered on the Larmor frequency, i.e., the $\Delta m= \pm 1$ transitions, $m=2 \leftrightarrow 1,1 \leftrightarrow 0,0 \leftrightarrow-1,-1 \leftrightarrow-2$. We collect spectra with both senses of nuclear spin polarization, i.e., the initial nuclear spin state is nearly either $|m=+2\rangle$ or $|-2\rangle$, so that the outer satellites, $( \pm 2 \leftrightarrow \pm 1)$ are dominant, and they are found on opposite sides of the central frequency in the two helicities. Examples of this include BCC Niobium ${ }^{28}$ and $\mathrm{SrTiO}_{3},{ }^{20}$ both cubic crystals where the interstitial site of the ${ }^{8} \mathrm{Li}^{+}$is noncubic with a finite electric field gradient (EFG) yielding a quadrupole split NMR. Among the FCC metals, we have never found a quadrupolar splitting for ${ }^{8} \mathrm{Li}$. This is strong evidence that the ${ }^{8} \mathrm{Li}^{+}$site has cubic symmetry in the FCC metals including $\mathrm{Pt}, \mathrm{Au}, \mathrm{Ag}$, and $\mathrm{Cu}$. The asymmetric resonance line shape in $\mathrm{Pd}$ is the same in both helicities (not shown), with a long tail towards zero shift, implying that even unresolved quadrupolar satellites cannot be the source of the shape. This is further confirmed by the broadening of the line with reduced temperature evident in Fig. 2. Quadrupolar effects, being determined by the surrounding lattice, are, in contrast, generally quite independent of temperature. A distribution of small EFGs at the cubic ${ }^{8} \mathrm{Li}^{+}$site from imperfections in the $\mathrm{Pd}$ lattice may give rise to unresolved quadrupolar splittings, but this must be, at most, a minor contribution to the line shape, or it would be evident as a difference between the two helicities.

In some FCC metals, we find ${ }^{8} \mathrm{Li}^{+}$spectra with two resonances, corresponding to two crystallographically distinct cubic sites in the lattice. As a function of temperature, there is a site change, meaning that either below or above some relatively narrow coexistence range, the spectrum consists of only one of these resonances. This is the case, for example, in both $\mathrm{Ag}$ and $\mathrm{Au},{ }^{21}$ where the low-temperature site is probably the octahedral interstitial site and the high-temperature site is the substitutional site. This site assignment is clearly confirmed by level crossing resonance in $\mathrm{Cu}{ }^{29}$ The sites are spectroscopically distinct because they have different hyperfine couplings and hence Knight shifts. For $\mathrm{Ag}, \mathrm{Au}$, and $\mathrm{Cu}$, the difference in hyperfine coupling is about a factor of 2 between the two sites with the high-temperature site having the lower coupling (and smaller positive shift). In contrast, in $\mathrm{Pd}$ and $\mathrm{Pt},{ }^{8}$ there is no evidence for a similar site change below $300 \mathrm{~K}$.

The broad high-frequency tail might, however, come from regions of the $\mathrm{Pd}$ that are somehow magnetically distinct, i.e., from magnetic inhomogeneity. We now consider two sources for such a magnetic inhomogeneity: strain in the Pd lattice, and proximity to the surface or substrate interface.

Strain is certainly expected to modify the magnetic properties of $\mathrm{Pd} .^{30}$ The lattice mismatch of $\mathrm{Pd}$ with the STO substrate is small $(0.4 \%)$ but finite, meaning that below a critical film thickness an epitaxial Pd film will be homogeneously expansively strained, while for thicker films the mismatch will be compensated by a network of misfit (edge type) dislocations. For sample IV, whose $100 \mathrm{~nm}$ thickness may exceed this critical thickness, dislocations will yield an inhomogeneous lattice strain within the film, yielding a continuous distribution of local strains depending on the distance of the ${ }^{8} \mathrm{Li}^{+}$probe to the dilute dislocation cores. However, the strain distribution should be substantially different in the polycrystalline film, which shows a similar spectrum. In the polycrystal film, rather than from the substrate, the strain is probably mainly due to the rapid low-temperature deposition (island growth mode) and is expected to be very different in both magnitude and 
distribution than in the epitaxial films. Thus, we regard strain as an unlikely source of the asymmetric line. It may, however, play a role in the thickness dependence of the shift (see Sec. V B).

Another possibility arises when the Pd line shape is compared to the NMR of small Pt particles. ${ }^{9}$ In intermediate size nanoparticles, where the NMR differs from the bulk, but is not yet completely dominated by the surface, the Pt NMR line shape is remarkably similar to our resonance in $\mathrm{Pd}$. The $\mathrm{Pt}$ line shape (in particles much smaller than our film thickness) is interpreted as a consequence of confinement effects on the free electrons, yielding a characteristic inhomogeneity on the atomic scale. Models based on an atomic layer dependent shift have been used to explain the complex particle size- and shape-dependent Pt NMR lines, see, e.g., Refs. 11,12, and 31. We will now apply this model to the simple geometry of a thin film, to determine if it can account for the observed line shape.

While no analogous data exist from Pd NMR, doubtless due to the low sensitivity of ${ }^{105} \mathrm{Pd}$, the magnetic properties of $\mathrm{Pd}$ nanoparticles have been interpreted using a model based on the Pt nanoparticle NMR. ${ }^{32}$ In this model, the electronic density of states at the Fermi level $\left(\rho_{0}\right)$ is postulated to heal exponentially back to the bulk value from a reduced value at the surface. The parameters of this healing are the exponential healing length $(0.4 \mathrm{~nm})$ and the amplitude of the surface reduction $(25 \%){ }^{32}$ A reduction in $\rho_{0}$, to $a \rho_{0}$ for $0<a<1$ affects the Stoner-enhanced magnetic susceptibility nonlinearly, via

$$
\chi=\chi_{0} \frac{a}{1-0.893 a},
$$

where the value 0.893 makes the Stoner enhancement agree with the bulk $\chi$ when $a=1$. To assess whether this model could yield the observed line shape, we implemented it for a thin film geometry including a depth-dependent sampling based on a truncated Gaussian representation of the implantation profile. To account for the surface resonance, we allow a surface layer of a defined thickness with a small positive shift. The field distribution from this model is convolved with a Lorentzian resolution function of width $1.8 \mathrm{kHz}$ (appropriate for the $\mathrm{CW}$ experiment) to calculate the model line shape. For sample IV, a negligible fraction of the ${ }^{8} \mathrm{Li}^{+}$ ions penetrate to the substrate interface, so we neglect its effect entirely in the model. Figure 8 shows the resulting profile of the inhomogeneous shift for two sets of parameters: those used to describe $\mathrm{Pd}$ nanoparticles ${ }^{32}$ and a set with a much larger healing length $\lambda$ and surface layer thickness that yields a line shape similar to the measured spectrum. In each case the surface reduction parameter of 0.25 and the Stoner enhancement of Eq. (5) was used. It is clear from the figure that the healing length from Ref. 32 is much too small to account for the line shape. A healing length about $15 \times$ as big is necessary to get a reasonable approximation to the line shape. Thus, the healing-length/surface-layer model can only be used to explain the line shape with parameters that are well outside the range used to explain the magnetic properties of $\mathrm{Pd}$ nanoparticles (or the NMR of $\mathrm{Pt}$ ). This seems quite unlikely, but we will come back to this model again to try to understand the thickness dependence in the next section.

While we have ruled out several possible origins of the asymmetric line as unlikely, we have also not yet found
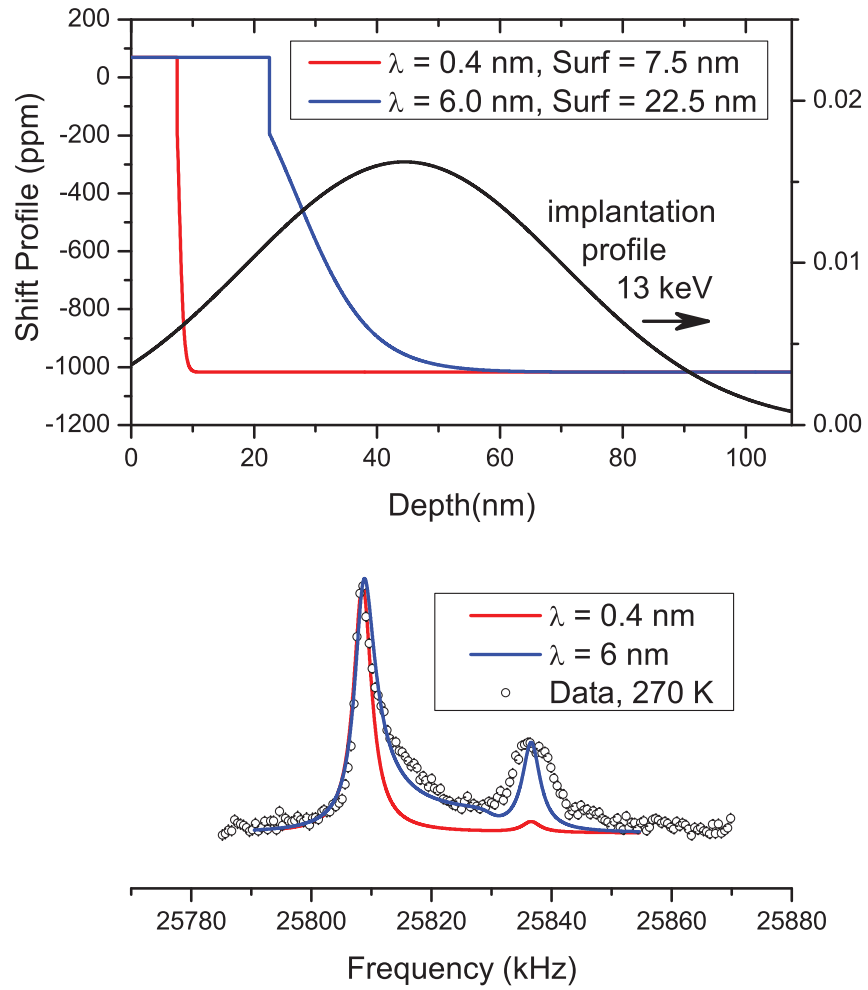

FIG. 8. (Color online) Top: Model depth profiles of the shift for the parameters used in Ref. 32 (red) and for a much larger healing length (blue). The surface layers are substantially different in thickness: $7.5 \mathrm{~nm}$ (red), corresponding to the Au capping layer thickness and $22.5 \mathrm{~nm}$ (blue), to yield an amplitude comparable to the observed surface resonance. The black curve shows a truncated Gaussian approximation of the $13 \mathrm{keV}$ implantation profile used to generate the corresponding lineshape in the bottom panel, where the colors correspond, and the circles show the experimental data.

a satisfactory explanation. More refined measurements will be required for this, but some hints may come from the sample dependence of the line shape that clearly contributes differently for the epitaxial films here (see Fig. 5), as well as the polycrystalline film ${ }^{6}$ and foil. ${ }^{23}$ From the current data, we can still draw important conclusions, largely from the position of the sharp peak of the Pd resonance (from the fits described in Sec. III) that is not sensitive to the detailed shape of the high-frequency tail.

\section{B. Effect of film thickness}

We turn now to the effect of thickness on the Pd shift. There is a clear reduction of the shift as thickness is reduced. The raw shift in the $30 \mathrm{~nm}$ films is reduced by about $20 \%$ compared to the $100 \mathrm{~nm}$ films (Fig. 9). This means the demagnetization field is also changing, and we cannot apply the same correction as above. Note the shift in $12.5 \mu \mathrm{m}$ thick Pd foil is comparable to the shift in the $100 \mathrm{~nm}$ film, so here we are justified in using the bulk $\chi$ to calculate the demagnetization field. Instead we plot the raw shifts in Fig. 9, and note,

$$
K=K_{c}-K_{d}=A \chi_{\mathrm{Li}}(T)-\frac{8 \pi}{3} \chi(T),
$$




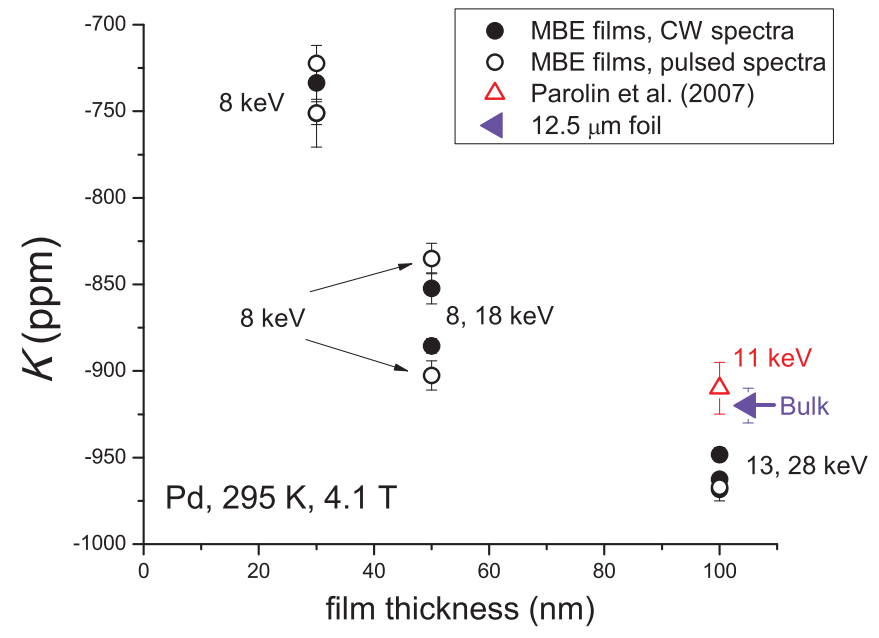

FIG. 9. (Color online) The Pd Knight shift in Pd at $295 \mathrm{~K}$ and 4.1 Tesla in MBE films of varying thickness in comparison with the polycrystalline film from Parolin et al. 6. The open(closed) circles correspond to the pulsed(CW) RF spectra. The pulsed spectra for samples I-III are all taken at $8 \mathrm{keV}$. The $\mathrm{CW}$ spectra were collected at $8 \mathrm{keV}$ and at $18 \mathrm{keV}$. For the $50 \mathrm{~nm}$ film (sample III) results from two energies are shown. Similarly for sample IV, the results for 13 and $28 \mathrm{keV}$ implantation energies are shown. While the energy dependence, measurement and analysis methods contribute to a scatter of the shift, the trend of a substantial reduction of shift with a reduction in film thickness is robust.

where $\chi_{\mathrm{Li}}(T)$ is the local spin susceptibility at the Li. At room temperature, $K_{c}$ scales with $\chi$, so $\chi_{\mathrm{Li}} \approx \chi$ with a clear deviation seen only below $100 \mathrm{~K}^{6}$

The dependence of the shift on thickness is well beyond the uncertainty in the shift determination and is clearly visible in the raw data. A large reduction in the magnitude of the shift was found previously in $e$-beam evaporated $\mathrm{Pd}$ films thinner than $100 \mathrm{~nm}^{24}$ However, irreproducibility and insufficient characterization of those samples suggested some uncontrolled factor in the fabrication (rather than thickness) was important. For this reason, we undertook the present measurements on very well-controlled films free of contamination from background gases and without significant strain from rapid growth.

With these much better controlled films, we now consider what might account for the thickness dependence. First we note the small expansive epitaxial strain, which should be most important in the thinnest films, would tend to make the Pd more magnetic, ${ }^{30}$ rather than less magnetic as we observe. Recall that conventional NMR in large assemblies of nanometer-sized Pt particles show asymmetric line broadening connected with electronic inhomogeneity near the surface. ${ }^{11}$ For Pt particles in the range $10 \mathrm{~nm}$ in diameter, the resonance corresponding to the bulk exhibits a reduction in the Knight shift. ${ }^{9}$ Given Pd's even greater sensitivity, it seems possible that the thickness dependence we observe in films of thickness several times this diameter may have a similar origin.

We explore this possibility further by applying the healing length model discussed in the previous section. Figure 10 shows candidate shift profiles for the $30 \mathrm{~nm}$ film at the $8 \mathrm{keV}$ implantation energy used. Note that at this energy, the ${ }^{8} \mathrm{Li}^{+}$is stopping in the vicinity of the buried interface
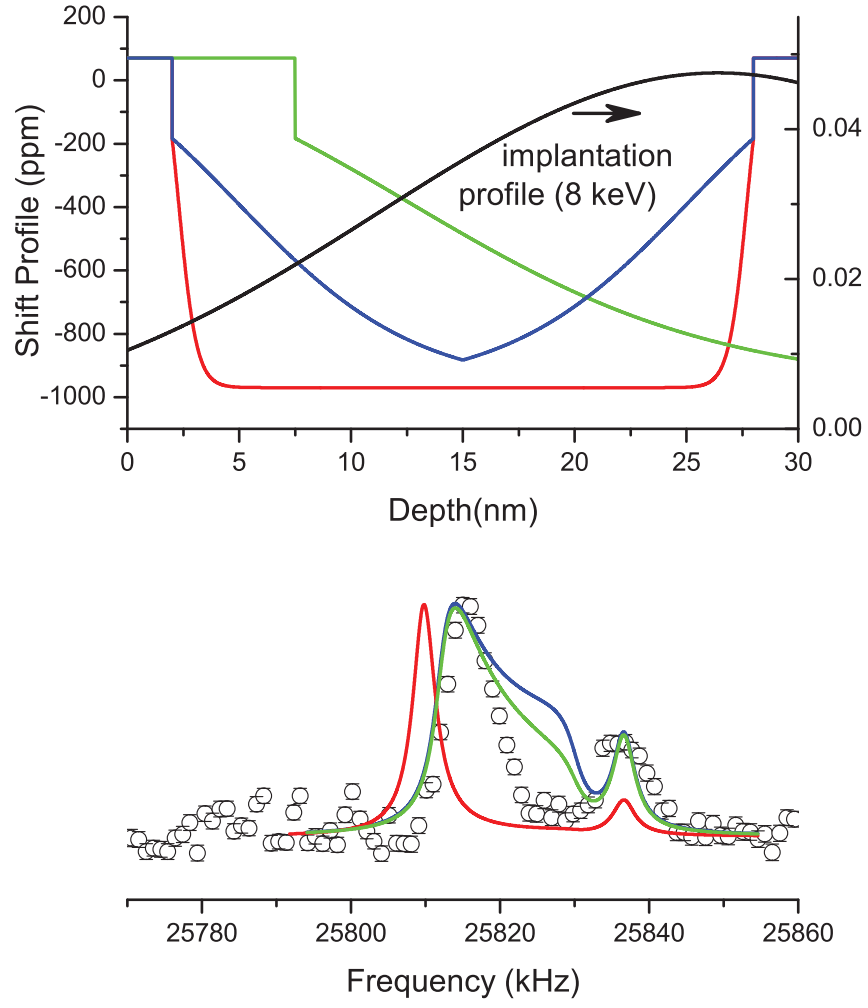

FIG. 10. (Color online) Top: Model depth profiles of the shift for the $30 \mathrm{~nm}$ thick film, for symmetric models that treat the surface and substrate interface as equivalent for the parameters used in Ref. 32 (red) and for a much larger healing lengths (blue) and (green) for a asymmetric model that neglects the substrate interface. The surface layers are substantially different in thickness: $2 \mathrm{~nm}$ (red, blue) and $7.5 \mathrm{~nm}$ (green), to yield a "surface" line comparable in amplitude to the observed spectrum. The black curve shows a truncated Gaussian model of the implantation profile used to generate the corresponding lineshape in the bottom panel, where the colors correspond and the points show experimental data.

with the STO substrate. First, it is clear that the healing length from Ref. 32 is unable to explain the reduction in shift (red profile and corresponding line shape in Fig. 10). Here we have also compared profiles with only a surface effect and profiles where the interface with the insulating substrate is treated equivalently to the free surface. An order of magnitude larger healing length can explain the reduction in shift, but the resulting line shape is much more asymmetric than the observed spectrum. Moreover, the profiles required to explain the reduced shift would predict a substantial implantation energy dependence of the resonance. In contrast, the energy dependence in sample IV (Fig. 11) is very weak. This inconsistency rules out the healing length phenomenon as the source of the thickness dependence and points toward a homogeneous reduction of the magnetic response as the $\mathrm{Pd}$ films are made thinner.

In ultrathin metal films, confinement effects lead to quantum well states that can certainly affect the density of states at the Fermi level, ${ }^{33}$ but such effects have only been seen in much thinner films (10s of monolayers, ML) while the thinnest films here $(30 \mathrm{~nm})$ are $180 \mathrm{ML}$ thick. However, a simple free electron estimate suggests the splitting between 


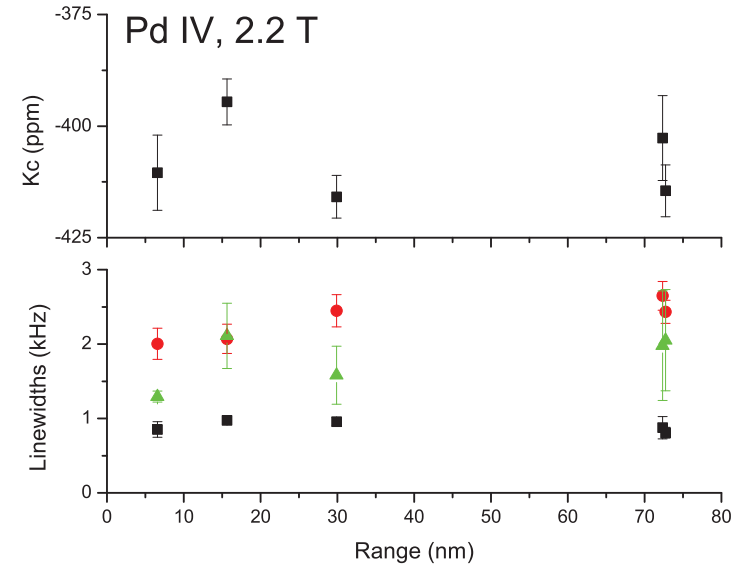

FIG. 11. (Color online) The ${ }^{8} \mathrm{Li}$ Knight shift in Pd at $295 \mathrm{~K}$ and 2.2 Tesla in sample IV as a function of the mean range of implantation as varied by the implantation energy. Bottom: The fitted resonance linewidths.

allowed $k_{z}$ states in the confining potential could still reach $\sim 100 \mathrm{meV}$ near $E_{F}$. In view of the narrow $d$ band at the Fermi level in $\mathrm{Pd}$, such a splitting may well substantially alter $\rho_{0}$, the Pauli susceptibility, and hence our shift. In contrast, existing calculations of Pd quantum wells find the opposite tendency towards ferromagnetism. ${ }^{34}$ On the other hand, a reduction in $\chi$ is more consistent with superconductivity found in some $\mathrm{Pd}$ films ${ }^{35}$ and the reduced $\chi$ found for some ligand-stabilized Pd nanoparticles and colloids, ${ }^{32}$ rather than with strain-induced ferromagnetism found in other $\mathrm{Pd}$ nanoparticles. ${ }^{36}$ The electron mean free path $l$ at $300 \mathrm{~K}$ in $\mathrm{Pd}$ is on the order of $10 \mathrm{~nm},{ }^{37}$ so the change in $\chi$ is occurring in films well into the three-dimensional regime where $l \ll t$, the thickness.

Another related possibility is a modification of the electronic structure of the films due to surface states. Surface states at (100) surfaces of $\mathrm{Pd}$ are known. ${ }^{38}$ Electrons populate this surface state, which is lower in energy than the bulk Fermi level over some fraction of the Fermi surface, thus reducing the Fermi level in the rest of the film. However, the surface state is highly localized to the top atomic layer and would not produce a large change in the electron distribution even in our thinnest films.

These results suggest that detailed electronic structure calculations for finite thickness Pd slabs (such as has been done for $\mathrm{Pt}^{39}$ ) should show interesting electronic and magnetic inhomogeneity. While less important than in $\mathrm{Pt}$, relativistic spin-orbit effects may also play a significant role.

\section{Temperature dependence of the Pd shift}

Now we consider the demagnetization corrected shift of the $\mathrm{Pd}$ resonance. In conventional NMR, where the probe nuclei are part of the lattice structure, one expects the Knight shift to be simply proportional to the spin susceptibility, as is found in Pd NMR. ${ }^{4,5}$ In a $d$-band metal, $K$ is usually decomposed into distinct contributions

$$
K_{c}=A_{d} \chi_{d}(T)+A_{s} \chi_{s}+K_{\mathrm{orb}},
$$

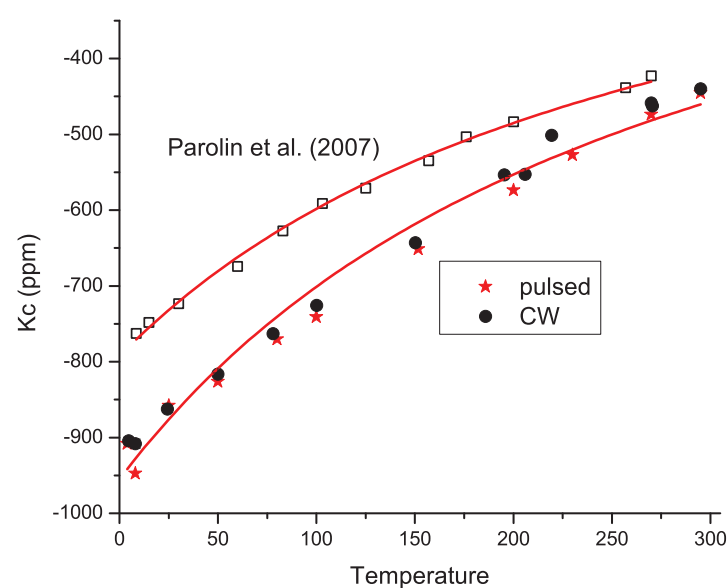

FIG. 12. (Color online) The temperature dependence of the Knight shift of ${ }^{8} \mathrm{Li}$ in two $\mathrm{Au}$ capped $100 \mathrm{~nm}$ films of $\mathrm{Pd}$, filled squares the polycrystalline film data of Ref. 6, open circles MBE sample IV. The data are fit to the Curie-Weiss dependence of the high temperature susceptibility of Pd extrapolated to low temperature.

where only the $d$-band spin susceptibility $\left(\chi_{d}\right)$ is assumed temperature dependent. $\chi_{s}$ is a Pauli-like temperature-independent term from the broad $s$ or $s p$ band, and $K_{\text {orb }}$ is the orbital or "chemical" shift. $A_{i}$ are the respective hyperfine couplings. Thus, we may expect that $K$ for ${ }^{8} \mathrm{Li}$ would follow

$$
K_{c}=A \chi(T)+B
$$

where $\chi(T)$ is the full susceptibility, which may also contain a $T$-independent orbital part. The constant $B$ accounts for all the temperature-independent terms. The value of $A$ here is about $-5 \mathrm{kG} / \mu_{B},{ }^{7}$ in comparison to $\mathrm{Pt}$, where it is about $-9 \mathrm{kG} / \mu_{B}{ }^{8}$

The implication of the plot in Fig. 12 is that the hyperfine field seen by the ${ }^{8} \mathrm{Li}$ nucleus does not simply scale with $\chi(T)$ below about $100 \mathrm{~K}$. Instead $K_{C}$ continues from room temperature to decrease almost linearly down to the lowest temperature. Such a simple dependence is remarkable. Motivated by our recent result in $\mathrm{Pt},{ }^{8}$ we find that the observed shift scales well with the high-temperature Curie-Weiss dependence of $\chi$ (see Fig. 6) extrapolated to low temperatures. With such a strong similarity to $\mathrm{Pt}$, this is not likely a coincidence, and it provides an important clue to the origin of the low-temperature deviations from Curie-Weiss $\chi$ in both these metals. The deviation from $\mathrm{C}-\mathrm{W}$ is eliminated by the presence of the probe ${ }^{8} \mathrm{Li}^{+}$, while the $\mathrm{C}-\mathrm{W}$ dependence itself survives. One calculation, based on the paramagnon model, ${ }^{40}$ attributes the maximum in $\chi(T)$ to a low-temperature $T^{2} \ln T$ term in $\chi$, and predicts that impurity scattering can remove this maximum, yielding an approximate $1 / T^{2}$ dependence at low T. Our result is partly consistent with this prediction, in that the maximum is removed and that $\chi$ continues to increase as $T$ is decreased. However, we do not find the predicted $1 / T^{2}$ behavior. In this picture, the ${ }^{8} \mathrm{Li}^{+}$provides the scattering that modifies $\chi$, so rather than the average $\chi$, our measurement reflects the local susceptibility at the scattering defect. This should be accounted for in a detailed comparison with theory. 


\section{Surface resonance}

In this section we discuss the surface resonance that occurs in both Au-capped and uncapped films. Its presence in previous measurements strongly suggest that it too is intrinsic to Pd. Its amplitude is enhanced by reducing the implantation energy (not shown), indicating it originates near the surface, but it is much too large to be due to the topmost atomic layers, as the accepted interpretation of the analogous line in the NMR of small Pt particles would suggest. Based on the behavior of the small-particle Pt NMR with intentional adsorbates, ${ }^{41}$ it is possible that adsorbates tie up the $d$ electrons of surface atoms in chemical bonds, removing them from the conduction band and strongly reducing the shift, but such an effect is not expected in the capped films, nor should it extend many ML into the interior of the film. Thus, it appears that other effects must conspire to yield a similar line, such as a confinement effect on the conduction electrons that is similar at both the free surface and the $\mathrm{Au} / \mathrm{Pd}$ interface.

The shift of the surface resonance is small, but clearly positive, and in the range of ${ }^{8} \mathrm{Li}$ Knight shifts in other metals that have no $d$ bands at the Fermi level. This suggests an alternative explanation: that the $d$-band susceptibility is strongly suppressed in the vicinity of the surface or the $\mathrm{Au} / \mathrm{Pd}$ interface, while a small $s$-band contribution remains, yielding a similar Knight shift to the other noble metals. Such an effect may be related to the surface state in $\mathrm{Pd}$, but its effect must extend over more than a few monolayers near the surface/interface to account for the size of the resonance.

\section{CONCLUSIONS}

We have studied the NMR properties of thin epitaxial films of Pd, a strongly exchange-enhanced, nearly magnetic transition metal with a large temperature-dependent susceptibility using implanted ${ }^{8} \mathrm{Li}^{+}$as a $\beta$-NMR probe. The large negative Knight shift is both temperature and thickness dependent. The spectra also contain a surface related line at small positive shift that is present in both Au-capped and bare films. The bifurcation into two types of Li site (Pd-like and surfacelike) suggests sharply inhomogeneous electronic properties as have been found in unsupported Pt particles. ${ }^{10}$ However, neither the Pd line shape nor the film thickness dependence of the shift can be explained using the healing length model used to describe conventional NMR in small metal particles. The observed thickness dependence of the Pd shift occurs in a range of thickness well above where quantum well confinement effects are known in thin metal films ${ }^{33}$ and are even significantly above estimates of the electron mean free path. The extreme sensitivity of Pd due to its very high density of states at the Fermi level, may, however, account for an enhanced sensitivity to confinement. Based on these results, we expect detailed calculations of the spatially and band-resolved electronic structure in finite thickness Pd slabs would exhibit novel properties, but we find no evidence of a tendency towards magnetism, as has been predicted for much thinner layers $^{34}$ and found in some Pd nanoparticles ${ }^{36}$ and possibly in hydrogen-coated $\mathrm{Pd}^{42}$ Instead, the reduction of shift in thinner films is at least qualitatively consistent with magnetic and NMR measurements in $\mathrm{Pt}^{9,11}$ and $\mathrm{Pd}^{32}$ nanoparticles.

This work opens a new avenue to study finite-size and confinement effects in metals with a depth-resolved local NMR probe where the sample geometry is much simpler and more controlled than with large ensembles of metallic nanoparticles. These remarkable results also suggest that $\beta$-NMR will be an important probe of correlated metals such as the transition metal oxides $\mathrm{Sr}_{2} \mathrm{RuO}_{4}$ and $\mathrm{LaNiO}_{3}$, the latter of which cannot be produced as a single crystal but can be made in highly controlled fashion as an epitaxial film with a metallic state that changes substantially as the films are made very thin.

\section{ACKNOWLEDGMENTS}

This work was supported by the Natural Sciences and Engineering Research Council (NSERC) Canada and CIfAR. W.A.M. acknowledges the hospitality of the Max Planck Institut für Festkörperforschung, Stuttgart during the final stages of this work. We thank M. Smadella for some help with the measurements, R. Abasalti, D. Arseneau, S. Daviel, B. Hitti, and D. Vyas for technical assistance, and A. Luther and L. J. de Jongh for useful discussions at an early stage of this work. We thank D. E. MacLaughlin for a critical reading of the manuscript.

\footnotetext{
*wam@chem.ubc.ca

${ }^{1}$ E.g., M. Nicklas, M. Brando, G. Knebel, F. Mayr, W. Trinkl, and A. Loidl, Phys. Rev. Lett. 82, 4268 (1999).

${ }^{2}$ E.g., H. Ackermann, P. Heitjans, and H.-J. Stöckmann, Top. Curr. Phys. 31, 291 (1983).

${ }^{3}$ G. D. Morris, W. A. MacFarlane, K. H. Chow, Z. Salman, D. J. Arseneau, S. Daviel, A. Hatakeyama, S. R. Kreitzman, C. D. P. Levy, R. Poutissou, R. H. Heffner, J. E. Elenewski, L. H. Greene, and R. H. Kiefl, Phys. Rev. Lett. 93, 157601 (2004).

${ }^{4}$ J. A. Seitchik, A. C. Gossard, and V. Jaccarino, Phys. Rev. 136, A1119 (1964).

${ }^{5}$ M. Takigawa and H. Yasuoka, J. Phys. Soc. Jpn. 51, 787 (1982).

${ }^{6}$ T. J. Parolin, Z. Salman, J. Chakhalian, Q. Song, K. H. Chow, M. D. Hossain, T. A. Keeler, R. F. Kiefl, S. R. Kreitzman, C. D. P. Levy, R. I. Miller, G. D. Morris, M. R. Pearson, H. Saadaoui, D. Wang, and W. A. MacFarlane, Phys. Rev. Lett. 98, 047601 (2007).
}

${ }^{7}$ T. J. Parolin, Ph.D. thesis, University of British Columbia, 2011, https://circle.ubc.ca/handle/2429/39778.

${ }^{8}$ O. Ofer, K. H. Chow, I. Fan, M. Egilmez, T. J. Parolin, M. D. Hossain, J. Jung, Z. Salman, R. F. Kiefl, C. D. P. Levy, G. D. Morris, M. R. Pearson, H. Saadaoui, Q. Song, D. Wang, and W. A. MacFarlane, Phys. Rev. B 86, 064419 (2012).

${ }^{9}$ H. E. Rhodes, P-K. Wang, H. T. Stokes, C. P. Slichter, and J. H. Sinfelt, Phys. Rev. B 26, 3559 (1982).

${ }^{10}$ I. Yu and W. P. Halperin, Phys. Rev. B 47, 15830 (1993).

${ }^{11}$ E.g., see the review: J. J. van der Klink and H. B. Brom, Prog. NMR Spectrosc. 36, 89 (2000).

${ }^{12}$ H. Yano, J. Inukai, H. Uchida, M. Watanabe, P. K. Babu, T. Kobayashi, J.-H. Chung, E. Oldfield, and A. Wieckowski, Phys. Chem. Chem. Phys. 8, 4932 (2006).

${ }^{13}$ T. Wagner, G. Richter, and M. Rühle, J. Appl. Phys. 89, 2606 (2001). 
${ }^{14}$ E.g., G. J. P. Abreu, R. Paniago, F. R. Negreiros, E. A. Soares, and H.-D. Pfannes, Phys. Rev. B 83, 165410 (2011).

${ }^{15}$ H. C. Jamieson and F. D. Manchester, J. Phys. F 2, 323 (1972).

${ }^{16}$ V. V. Sobolev, D. O. Mordas, and V. V. Sobolev, Glass Phys. Chem. 29, 360 (2003).

${ }^{17}$ K. von Rottkay, M. Rubin, and P. A. Duine, J. Appl. Phys. 85, 408 (1999).

${ }^{18}$ H. Saadaoui, G. D. Morris, Z. Salman, Q. Song, K. H. Chow, M. D. Hossain, C. D. P. Levy, T. J. Parolin, M. R. Pearson, M. Smadella, D. Wang, L. H. Greene, P. J. Hentges, R. F. Kiefl, and W. A. MacFarlane, Phys. Rev. B 83, 054504 (2011).

${ }^{19} \mathrm{~W}$. Eckstein, Computer Simulation of Ion-Solid Interactions (Springer, Berlin, 1991).

${ }^{20}$ W. A. MacFarlane, G. D. Morris, K. H. Chow, R. A. Baartman, S. Daviel, S. R. Dunsiger, A. Hatakeyama, S. R. Kreitzman, C. D. P. Levy, R. I. Miller, K. M. Nichol, R. Poutissou, E. Dumont, L. H. Greene, and R. F. Kiefl, Physica B 326, 209 (2003).

${ }^{21}$ T. J. Parolin, Z. Salman, K. H. Chow, Q. Song, J. Valiani, H. Saadaoui, A. O'Halloran, M. D. Hossain, T. A. Keeler, R. F. Kiefl, S. R. Kreitzman, C. D. P. Levy, R. I. Miller, G. D. Morris, M. R. Pearson, M. Smadella, D. Wang, M. Xu, and W. A. MacFarlane, Phys. Rev. B 77, 214107 (2008).

${ }^{22}$ B. Ittermann, G. Welker, F. Kroll, F. Mai, K. Marbach, and D. Peters, Phys. Rev. B 59, 2700 (1999).

${ }^{23}$ T. J. Parolin, Z. Salman, J. Chakhalian, D. Wang, T. A. Keeler, M. D. Hossain, R. F. Kiefl, K. H. Chow, G. D. Morris, R. I. Miller, and W. A. MacFarlane, Physica B 374-375, 419 (2006).

${ }^{24}$ T. J. Parolin, Z. Salman, J. Chakhalian, Q. Song, K. H. Chow, G. D. Morris, M. Egilmez, I. Fan, M. D. Hossain, T. A. Keeler, R. F. Kiefl, S. R. Kreitzman, C. D. P. Levy, A. I. Mansour, R. I. Miller, M. R. Pearson, H. Saadaoui, M. Smadella, D. Wang, M. Xu, and W. A. MacFarlane, Physica B 404, 917 (2009).

${ }^{25}$ M. Xu, M. D. Hossain, H. Saadaoui, T. J. Parolin, K. H. Chow, T. A. Keeler, R. F. Kiefl, G. D. Morris, Z. Salman, Q. Song, D. Wang, and W. A. MacFarlane, J. Magn. Res. 191, 47 (2008).

${ }^{26}$ The data included are from W. D. Weiss and R. Kohlhaas, Z. Angew. Phys. 23, 175 (1967) and Refs. 4, 15.

${ }^{27}$ G. C. Carter, L. H. Bennett, and D. J. Kahan, Metallic Shifts in NMR, Progress in Materials Science, Vol. 20, edited by B. Chalmers, J. W. Christian, and T. B. Massalski (Pergamon Press, Oxford, 1977), Pt. 1.
${ }^{28}$ T. J. Parolin, J. Shi, Z. Salman, K. H. Chow, P. Dosanjh, H. Saadaoui, Q. Song, M. D. Hossain, R. F. Kiefl, C. D. P. Levy, M. R. Pearson, and W. A. MacFarlane, Phys. Rev. B 80, 174109 (2009).

${ }^{29}$ K. H. Chow, A. I. Mansour, I. Fan, R. F. Kiefl, G. D. Morris, Z. Salman, T. Dunlop, W. A. MacFarlane, H. Saadaoui, O. Mosendz, B. Kardasz, B. Heinrich, J. Jung, C. D. P. Levy, M. R. Pearson, T. J. Parolin, D. Wang, M. D. Hossain, Q. Song, and M. Smadella, Phys. Rev. B 85, 092103 (2012).

${ }^{30}$ T. Jarlborg and A. J. Freeman, Phys. Rev. B 23, 3577 (1981); A. Hjelm, Int. J. Mod. Phys. B 7, 275 (1993); E. Hüger and K. Osuch, Eur. Phys. J. B 44, 145 (2005); H. Chen, N. E. Brener, and J. Callaway, Phys. Rev. B 40, 1443 (1989); V. L. Moruzzi and P. M. Marcus, ibid. 39, 471 (1989); L. Fritsche, J. Noffke, and H. Eckardt, J. Phys. F 17, 943 (1987); S. C. Hong and J. I. Lee, J. Korean Phys. Soc. 52, 1099 (2008).

${ }^{31}$ J. P. Bucher, J. Buttet, J. J. van der Klink, and M. Graetzel, Surf. Sci. 214, 347 (1989).

${ }^{32}$ V. Volokitin, J. Sinzig, G. Schmid, H. Bönnemann, and L. J. de Jongh, Z. Phys. D 40, 136 (1997); D. A. van Leeuwen, J. M. van Ruitenbeek, G. Schmid, and L. J. de Jongh, Phys. Lett. A 170, 325 (1992).

${ }^{33}$ T.-C. Chiang, Surf. Sci. Rep. 39, 181 (2000).

${ }^{34}$ S. Mirbt, B. Johansson, and H. L. Skriver, Phys. Rev. B 53, R13310 (1996); S. C. Hong, J. I. Lee, and R. Wu, ibid. 75, 172402 (2007).

${ }^{35}$ E.g., A. Cosceev, C. Sürgers, H.-G. Boyen, P. Schweiss and H. v. Löhneysen, Phys. Rev. B 83, 174516 (2011).

${ }^{36}$ Y. Oba, T. Sato, and T. Shinohara, Phys. Rev. B 78, 224417 (2008).

${ }^{37}$ R. S. Markiewicz and C. J. Rollins, Phys. Rev. B 29, 735 (1984).

${ }^{38}$ O. Rader, E. Vescovo, J. Redinger, S. Blügel, C. Carbone, W. Eberhardt, and W. Gudat, Phys. Rev. Lett. 72, 2247 (1994).

${ }^{39}$ M. Weinert and A. J. Freeman, Phys. Rev. B 28, 6262 (1983).

${ }^{40}$ D. R. Grempel, Phys. Rev. B 27, 4281 (1983).

${ }^{41}$ E.g., P.-K. Wang, J.-P. Ansermet, S. L. Rudaz, Z. Wang, S. Shore, C. P. Slichter, and J. H. Sinfelt, Science 234, 35 (1986).

${ }^{42}$ P. Amann, M. Cordin, J. Redinger, S. D. Stolwijk, K. Zumbrägel, M. Donath, E. Bertel, and A. Menzel, Phys. Rev. B 85, 094428 (2012). 